\title{
Risankizumab in patients with moderate to severe Crohn's disease: an open-label extension study
}

\author{
Brian G Feagan, Julián Panés, Marc Ferrante, Arthur Kaser, Geert R D'Haens, William J Sandborn, Edouard Louis, Markus F Neurath, \\ Denis Franchimont, Olivier Dewit, Ursula Seidler, Kyung-Jo Kim, Christian Selinger, Steven J Padula, Ivona Herichova, Anne M Robinson, \\ Kori Wallace, Jun Zhao, Mukul Minocha, Ahmed A Othman, Adina Soaita, Sudha Visvanathan, David B Hall, WulfO Böcher
}

\section{Summary}

Background Risankizumab, an anti-interleukin 23 antibody, was superior to placebo in achieving clinical and endoscopic remission at week 12 in a randomised, phase 2 induction study in patients with moderately to severely active Crohn's disease. Here we aimed to assess the efficacy and safety of extended intravenous induction and subcutaneous maintenance therapy with risankizumab.

Methods All patients who completed the 12-week induction phase of the double-blind phase 2 induction study were included in this open-label extension study. Patients who did not achieve deep remission, defined as clinical remission (Crohn's Disease Activity Index [CDAI] $<150$ ) and endoscopic remission (Crohn's Disease Endoscopic Index of Severity $[$ CDEIS] $\leq 4$, or $\leq 2$ for patients with isolated ileitis), at week 12 received open-label intravenous therapy with $600 \mathrm{mg}$ risankizumab every 4 weeks for 12 weeks; patients in deep remission at week 12 entered a 12-week washout phase. Patients in clinical remission at week 26 were invited to participate in the maintenance phase of the study, in which they received open-label subcutaneous risankizumab $(180 \mathrm{mg})$ every 8 weeks for 26 weeks. 26-week efficacy endpoints were the proportion of patients in clinical remission (CDAI $<150$ ), and the proportion of patients who achieved clinical response (either CDAI of $<150$ or a reduction from baseline of at least 100 points). 52-week efficacy endpoints were the proportion of patients achieving: clinical remission; clinical response; endoscopic response (>50\% CDEIS reduction from baseline); endoscopic remission, as defined previously; mucosal healing; and deep remission. Safety was assessed in patients who received at least one dose of the study drug during the open-label phases of the study. This study is registered with ClinicalTrials.gov, number NCT02031276.

Findings Of the 108 patients who completed the 12-week double-blind induction trial, six patients were in deep remission and entered the 12-week washout phase. 102 patients were not in deep remission, 101 of whom received 12 weeks of $600 \mathrm{mg}$ risankizumab (33 from the original placebo group, 34 from the $200 \mathrm{mg}$ risankizumab group, and 34 from the $600 \mathrm{mg}$ risankizumab group); the other patient declined to continue the study. At week 26, 54 (53\%) of 101 patients treated with $600 \mathrm{mg}$ rizankizumab were in clinical remission. Among patients included in the open-label extension trial, clinical remission rates at week 26 versus week 12 were: $18(55 \%)$ versus six (18\%) of 33 patients in the original placebo group; 20 (59\%) versus seven (21\%) of 34 patients in the original $200 \mathrm{mg}$ risankizumab group; and $16(47 \%)$ versus nine (26\%) of 34 patients in the original $600 \mathrm{mg}$ risankizumab group. 62 patients received risankizumab maintenance treatment, including the 54 patients who achieved clinical remission at week 26, the six patients who had achieved deep remission at week 12, and one patient because of a protocol violation. At week 52 , clinical remission was maintained in $44(71 \%)$ patients; $50(81 \%)$ patients had a clinical response, $22(35 \%)$ patients were in endoscopic remission, and 34 (55\%) patients had an endoscopic response. 15 (24\%) patients had mucosal healing and $18(29 \%)$ patients achieved deep remission at week 52 . Risankizumab was well tolerated with no new safety signals noted. The most frequent treatment-emergent adverse events were arthralgia (25 [22\%] of 115 patients), headache (23 [20\%]), abdominal pain $(21[18 \%])$, nasopharyngitis $(18[16 \%])$, nausea $(18$ [16\%]), and pyrexia (15 [13\%]). Most adverse events were mild or moderate and considered to be unrelated to study treatment. There were no treatment-related deaths.

Interpretation Extended induction treatment with open-label intravenous risankizumab was effective in increasing clinical response and remission rates at week 26. Open-label subcutaneous risankizumab maintained remission until week 52 in most patients who were in clinical remission at week 26. Selective blockade of interleukin 23 warrants further investigation as a treatment for Crohn's disease.

Funding Boehringer Ingelheim.

Copyright (C) 2018 Elsevier Ltd. All rights reserved.

\section{Introduction}

Crohn's disease is a life-long, relapsing, remitting inflammatory disease of the gastrointestinal tract with symptoms of abdominal pain, weight loss, and chronic diarrhoea. ${ }^{1,2}$ The medical management of Crohn's disease is based on the use of corticosteroids and

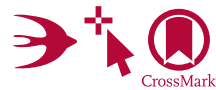

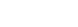

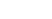


Boehringer Ingelheim Pharmaceuticals Inc, Ridgefield, CT, USA (A Soaita PhD, S Visvanathan PhD, D B Hall PhD) Correspondence to: Dr Brian G Feagan, Western University, Robarts Clinical Trials, London, ON N6A 5B6, Canada brian.feagan@robartsinc.com

\section{Research in context}

\section{Evidence before this study}

We searched PubMed for English language articles using the terms "Crohn's disease", "biologic therapy", "adalimumab", "infliximab", "certolizumab pegol", "vedolizumab",

"ustekinumab", and "IL-23" to identify controlled clinical trials published up to March 28, 2018, with no start date restrictions. In the USA and Europe, current biological therapies approved for the treatment of moderate-to-severe Crohn's disease include the tumour necrosis factor (TNF) antagonists adalimumab, infliximab, and certolizumab pegol, the integrin antagonists vedolizumab and natalizumab (USA only), and the interleukin 12 and interleukin 23 inhibitor, ustekinumab. Treatment regimens are aimed at inducing remission, followed by maintenance therapy. Despite several therapeutic options, there are a number of patients in whom these therapies have limited efficacy (primary non-response), who lose response over time (secondary non-response) or develop adverse effects; thus, new therapies targeting different inflammatory pathways are needed. Previously, we reported phase 2 clinical trial results of induction treatment with two intravenous doses of risankizumab $(200 \mathrm{mg}$ and $600 \mathrm{mg}$ every 4 weeks), a monoclonal antibody targeting the interleukin $23 \mathrm{p} 19$ subunit, in treatment-experienced patients with moderate-to-severe Crohn's disease. Risankizumab was superior to placebo in achieving clinical response or remission at week 12 , demonstrating proof of concept.

\section{Added value of this study}

This study reports findings from a 12 -week open-label

intravenous induction phase of the trial, in which patients not in deep remission after the first 12 weeks of induction treatment were given $600 \mathrm{mg}$ intravenous risankizumab every 4 weeks for 12 weeks; and a 26 -week open-label maintenance phase, in which patients in deep clinical remission at week 26 were treated with $180 \mathrm{mg}$ subcutaneous risankizumab every 8 weeks for 26 weeks. Extended, open-label induction treatment with $600 \mathrm{mg}$ intravenous risankizumab resulted in greater clinical remission rates than those observed at week 12, among patients not in deep remission at week 12 . Maintenace therapy with subcutaneous risankizumab (180 mg) was effective in maintaining clinical remission up to week 52 in patients who were in clinical remission at week 26, although a placebo group was not included for comparison in either phase of this open-label extension study.

\section{Implications of all the available evidence}

Evidence from studies with other biological therapies suggests that some patients, particularly those previously exposed to TNF antagonists, can take longer to achieve remission than patients naive to TNF antagonists. 93\% of patients enrolled in this study had been previously treated with at least one TNF antagonist and $79 \%$ had failed at least one such treatment because of inadequate response, loss of response, or intolerance. On the basis of these results, specific blockade of interleukin 23 with risankizumab appears to be a promising new approach for the induction of response and maintenance treatment of Crohn's disease, and is currently undergoing phase 3 investigation. immunosuppressive agents such as thiopurines or methotrexate, with the aim of controlling mucosal inflammation and inducing clinical remission. ${ }^{1,3}$ Although corticosteroids are successful for induction of remission, they are ineffective as maintenance therapy and are associated with an increased risk of adverse events, such as serious infection. ${ }^{1}$ The advent of biological therapies that selectively target cytokines or integrins has provided alternative treatment options for patients with moderate-to-severe Crohn's disease; however, these therapies are not universally effective, can lose effectiveness over time, and might also predispose patients to infections. ${ }^{1,46}$

Interleukin 23 plays a key part in the induction and function of immune cells, including T-helper 17 cells, innate lymphoid cells, $\gamma \delta$ T cells, and natural killer cells, which are responsible for tissue inflammation, destruction, and aberrant tissue repair that underlies the pathology of several immune-related disorders, including Crohn's disease. ${ }^{7-12}$ Furthermore, polymorphisms in the interleukin 23 receptor gene are associated with susceptibility to both Crohn's disease and ulcerative colitis. ${ }^{7}$ Blockade of the interleukin 23 pathway using biological agents that target interleukin 23 (guselkumab, risankizumab, tildrakizumab), the downstream cytokine interleukin 17 or its receptor (brodalumab, ixekizumab, secukinumab), or interleukin 12 and interleukin 23 simultaneously (ustekinumab) are effective for the treatment of psoriasis; risankizumab and ustekinumab have also shown efficacy in patients with Crohn's disease. ${ }^{13-16}$ Conversely, biological therapies that specifically target interleukin 17 or the interleukin 17 receptor exacerbate Crohn's disease, indicating differences between these cytokine pathways in the pathophysiology of psoriasis compared with that of Crohn's disease. ${ }^{17-19}$

Risankizumab (BI 655066/ABBV-066) is a humanised monoclonal IgG1 antibody targeting the interleukin 23 p19 subunit, ${ }^{20}$ currently under investigation in Crohn's disease, psoriasis, psoriatic arthritis, and asthma. In a randomised, double-blind, phase 2 study $^{21}$ in patients with moderately to severely active Crohn's disease, most of whom had previously received at least two tumour necrosis factor (TNF) antagonists, intravenous induction therapy with risankizumab (200 $\mathrm{mg}$ or $600 \mathrm{mg}$ at weeks 0,4 , and 8 ) was superior to placebo in achieving clinical remission and endoscopic remission at week 12, based on a pooled analysis of the two drug groups. The greatest 
treatment response was achieved with $600 \mathrm{mg}$ risankizumab, which resulted in clinical remission for $37 \%$ of patients compared with $15 \%$ of patients receiving placebo (difference 20.9\%; $\mathrm{p}=0 \cdot 025$ ). ${ }^{21}$ Since evidence from studies with other biological therapies suggests that some patients, particularly those with extensive previous treatment with TNF antagonists, can take longer to achieve remission, ${ }^{1,22}$ this study included a prespecified 12 -week open-label induction phase of treatment with $600 \mathrm{mg}$ intravenous risankizumab every 4 weeks for patients who did not achieve deep remission at week 12; followed by a 26-week open-label maintenance phase of treatment with subcutaneous $180 \mathrm{mg}$ risankizumab every 8 weeks for patients in clinical remission at week 26. We report the safety and additional efficacy endpoints from these open-label treatment periods.

\section{Methods}

\section{Study design and participants}

A complete description of the inclusion and exclusion criteria has been reported previously. ${ }^{21}$ Briefly, eligible patients were adults (aged 18-75 years) who had been diagnosed with Crohn's disease for at least 3 months and who had moderate to severe symptoms at screening, defined by a Crohn's Disease Activity Index (CDAI) ${ }^{23}$ of 220-450, with mucosal ulcers in the ileum or colon (or both), and a Crohn's Disease Endoscopic Index of Severity (CDEIS) ${ }^{23}$ of at least 7 (or $\geq 4$ for patients with isolated ileitis) on ileocolonoscopy, scored by a masked central reader. In the double-blind phase of the study (weeks 0-12; Period 1), participants received one of two doses of risankizumab or placebo for 12 weeks as induction therapy. In this open-label extension phase of the study, patients not in deep remission at week 12 were given extended induction therapy with intravenous risankizumab (600 mg) every 4 weeks for 12 weeks, and patients who achieved deep remission at week 12 entered a washout phase. Those in clinical remission at week 26 were treated with open-label subcutaneous risankizumab as maintenance therapy for 26 weeks. The efficacy analysis population comprised all patients who received at least one dose of study drug in the open-label induction or maintenance phases of the study.

The study protocol was approved by the institutional review board or ethics committee at each participating centre. Safety data were periodically assessed by an independent data monitoring committee. Written informed consent was provided by all patients.

\section{Procedures}

In the double-blind phase of the study (weeks 0-12; Period 1), patients received either risankizumab $200 \mathrm{mg}$, risankizumab $600 \mathrm{mg}$, or placebo by intravenous infusion at weeks 0,4 , and $8 .{ }^{21}$ Patients who were not in deep remission, defined as clinical and endoscopic remission, at week 12 received open-label risankizumab $600 \mathrm{mg}$ intravenous infusion at weeks 14, 18, and 22 (weeks 14-26; Period 2). Patients who were in deep remission at week 12 entered a washout phase until week 26. If these patients experienced a disease flare during this period (including the week 26 visit), defined as an increase in CDAI of at least 70 points compared with week 12, and a CDAI of at least 220, patients had an ileocolonoscopy. If the CDEIS was 4 or less (or $\leq 2$ for patients with initial isolated ileitis), patients continued with washout phase until week 26; otherwise they were to commence open-label intravenous infusions of $600 \mathrm{mg}$ risankizumab every 4 weeks for 12 weeks. At week 26, patients who were not in clinical remission stopped the study. Patients who were in clinical remission at week 26 were given the option to receive maintenance therapy with $180 \mathrm{mg}$ subcutaneous risankizumab at weeks 26, 34, 42, and 50 (weeks 26-52; Period 3). We selected the maintenance dosing regimen on the basis of the pharmacokinetics and available formulation of risankizumab, and the clinical results observed in patients with plaque psoriasis, suggesting an extended clinical effect and the expectation of higher drug clearance in patients with Crohn's disease. ${ }^{24}$

\section{Outcomes}

The primary efficacy endpoint of phase 1 (ie, the proportion of patients in clinical remission, defined by a CDAI of $<150$ at week 12 , in the intention-to-treat population), has been previously reported. ${ }^{21}$ 26-week efficacy outcomes were the proportion of patients in clinical remission (CDAI of <150) and the proportion of patients who achieved clinical response (CDAI of $<150$ or a reduction from baseline of at least 100 points). Endoscopy was not done at week 26. 52-week efficacy outcomes were the proportion of patients who achieved clinical remission, clinical response, endoscopic remission (CDEIS $\leq 4$ or $\leq 2$ for patients with baseline-isolated ileitis), endoscopic response ( $>50 \%$ CDEIS reduction from baseline), mucosal healing (absence of mucosal ulceration), and deep remission (clinical remission plus endoscopic remission). Exploratory endpoints were serum C-reactive protein, faecal calprotectin, and faecal lactoferrin concentrations.

During Periods 2 and 3, the CDAI was assessed at every treatment visit as well as at the end of each period (weeks 26 and 52). Differences in the visit windows used for analysis during open-label extension and the blinded period meant there was a discrepancy with the previous study in the number of patients reported to be in clinical remission at week 12 in the $200 \mathrm{mg}$ group. CDEIS was assessed by a masked and independent central reader at week 52. C-reactive protein, faecal calprotectin, and faecal lactoferrin were assessed at weeks 18, 26, 34, 50, and 52. Plasma samples were collected at every study visit and were used to determine risankizumab concentrations and to assess the immunogenicity of risankizumab. Assay methods have been previously described. ${ }^{21}$ 
Articles

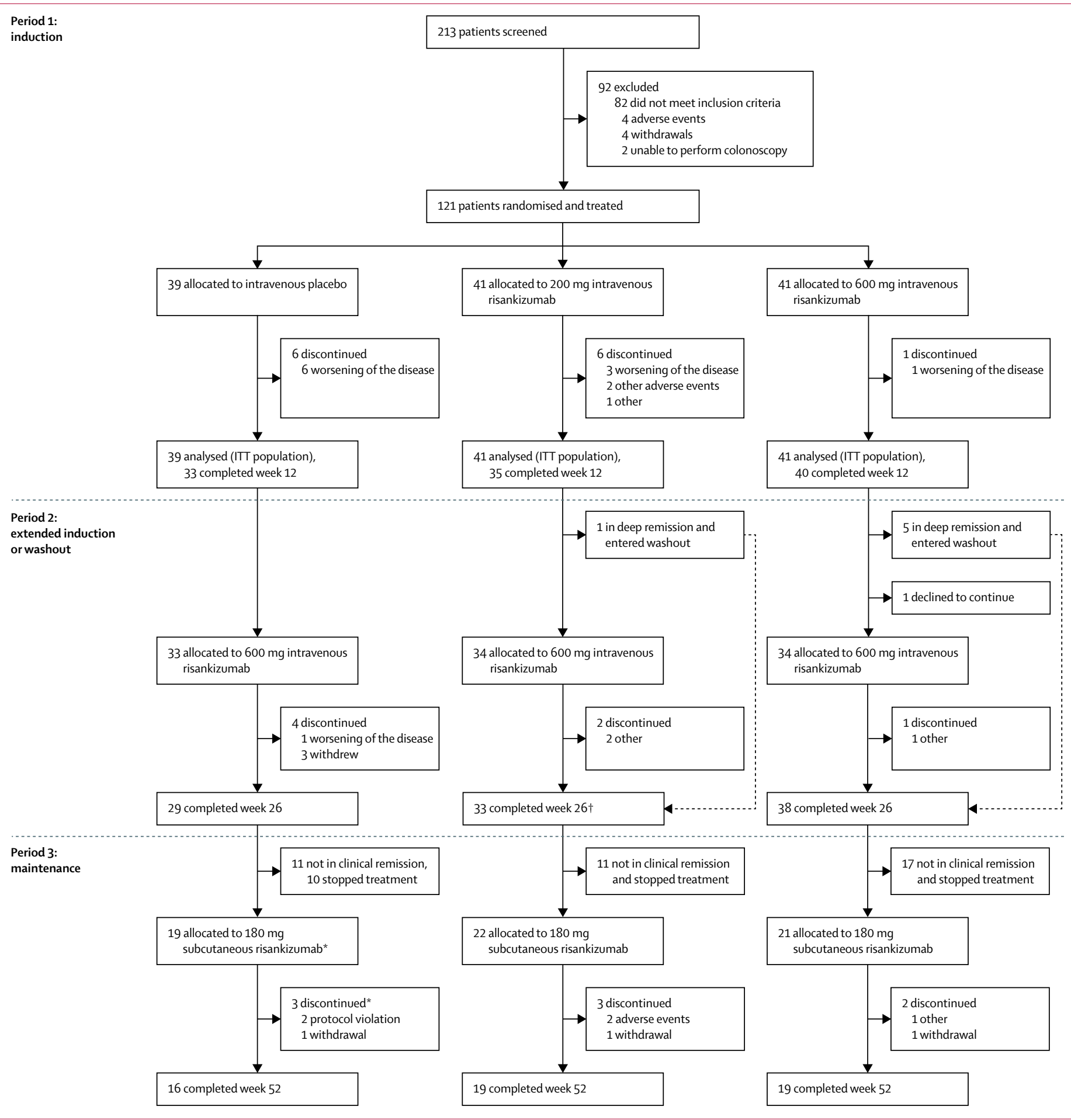

\section{Figure 1: Trial profile}

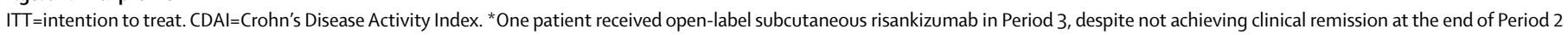

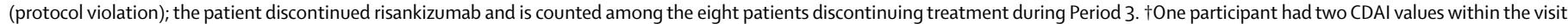
window for week 26; the value used for the efficacy calculation (CDAI of 166) differed from the value used to determine eligibility for entry into Period 3 (CDAI of 110 ). 
Safety endpoints were adverse events, serious adverse events, tolerability, changes in vital signs and physical examination, discontinuation of therapy because of adverse events, laboratory assessments at all study visits, and 12-lead electrocardiogram at weeks 26 and 52.

\section{Statistical analysis}

Endpoints for Periods 2 and 3 were summarised descriptively, unless specified. The efficacy analysis population for Periods 2 and 3 included patients who received at least one dose of $600 \mathrm{mg}$ intravenous risankizumab during the relevant study period); patients who were in washout in Period 2 were not included. For longitudinal analyses, we used last observation carried forward for missing values and stratified CochranMantel-Haenszel tests. For analysis of binary endpoints, we used non-response imputation for missing values. We used post-hoc, stepwise selection logistic regression analyses to explore the predictive potential of baseline factors (CDAI, duration of disease, abdominal pain, stool frequency, corticosteroids use, TNF antagonist use, presence of draining fistulas, and disease location) for week 52 clinical or endoscopic remission or response. We did these post-hoc regression analyses of the efficacy analysis population of Period 3 using the Period 1 treatment group assignment as one factor. A significance level of 0.05 was set for step 1 entry into the model.

We estimated the incidence of treatment-emergent adverse events per 100 patient-years for exposure to $600 \mathrm{mg}$ intravenous risankizumab during Period 2 (in the efficacy analysis population), $180 \mathrm{mg}$ subcutaneous risankizumab during Period 3 (in the efficacy analysis population), and for all patients exposed to risankizumab during Periods 1-3 combined (all-exposure safety population).

This trial is registered with ClinicalTrials.gov, number NCT02031276.

\section{Role of the funding source}

The funder of the study had a role in study design, data collection, data analysis, data interpretation, and writing of the report. The corresponding author had full access to all the data in the study and had final responsibility for the decision to submit for publication.

\section{Results}

Of the 108 patients who completed the 12-week double-blind induction trial, six patients were in deep remission and entered the 12-week washout phase. 102 patients were not in deep remission, 101 of whom received 12 weeks of $600 \mathrm{mg}$ risankizumab (33 from the original placebo group, 34 from the $200 \mathrm{mg}$ risankizumab group, and 34 from the $600 \mathrm{mg}$ risankizumab group); the other patient declined to continue the study (figure 1). The baseline demographics and disease characteristics of patients who participated in the open-label extension and maintenance phases of the study were similar across treatment groups (table 1). ${ }^{21}$ The mean duration of Crohn's disease at study entry was 14 years (SD 10). Of the 121 patients randomly assigned in Period 1, 115 received at least one dose of risankizumab during all three study periods (all-exposure safety population). 107 patients continued to Period 2 (13 patients discontinued during Period $1^{21}$ and one patient in the original $600 \mathrm{mg}$ risankizumab group declined to continue participation).

The proportion of patients who received risankizumab in Period 2 with clinical response or remission increased steadily from week 14 to week 26 (figure 2A). The proportion of patients with clinical remission at week 26 was greater than that observed for the same patients at week 12 (table 2): 18 (55\%) of 33 patients at week 26 versus six $(18 \%)$ of 33 patients at week 12 , for the original placebo group; 20 (59\%) of 34 versus seven (21\%) of 34 for the $200 \mathrm{mg}$ risankizumab group; and 16 (47\%) of 34 versus nine (26\%) of 34 for the $600 \mathrm{mg}$ risankizumab group (appendix $\mathrm{p}$ 4). Seven patients discontinued $600 \mathrm{mg}$ risankizumab during Period 2 because of patient withdrawal $(n=3)$, worsening disease $(n=1)$, and lack of efficacy $(n=3)$. Mean CDAI decreased from week 12 to week 26 (figure 2B).

62 patients were in clinical remission at week 26 and commenced maintenance therapy with $180 \mathrm{mg}$ subcutaneous risankizumab. Of these patients, 55 were in clinical remission at week 26 after receiving treatment in Period 2 (including the patient with discrepant CDAI scores in the $200 \mathrm{mg}$ group), six had been in deep

\begin{tabular}{|c|c|c|c|c|}
\hline & \multirow[t]{2}{*}{ Placebo $(n=33)$} & \multicolumn{2}{|l|}{ Risankizumab } & \multirow[t]{2}{*}{ All $(n=107)$} \\
\hline & & $200 \mathrm{mg}(\mathrm{n}=35)$ & $600 \mathrm{mg}(\mathrm{n}=39)$ & \\
\hline Mean age, years & $36(14)$ & $39(13)$ & $40(13)$ & $39(13)$ \\
\hline \multicolumn{5}{|l|}{ Sex } \\
\hline Female & $19(58 \%)$ & $20(57 \%)$ & $23(59 \%)$ & $62(58 \%)$ \\
\hline Male & $14(42 \%)$ & $15(43 \%)$ & $16(41 \%)$ & $45(42 \%)$ \\
\hline Duration of disease, years & $12(10)$ & $15(9)$ & $14(10)$ & $14(10)$ \\
\hline CDAI & $287(246-365)$ & $311(258-374)$ & $298(246-330)$ & $297(246-358)$ \\
\hline CDEIS & $11(8-18)$ & $12(9-16)$ & $12(8-16)$ & $12(9-17)$ \\
\hline $\mathrm{CRP}, \mathrm{mg} / \mathrm{L}$ & $10(3-24)$ & $10(4-29)$ & $8(2-29)$ & $10(3-29)$ \\
\hline \multicolumn{5}{|l|}{ Disease site } \\
\hline Ileum only & $3(9 \%)$ & $4(11 \%)$ & $6(15 \%)$ & $13(12 \%)$ \\
\hline Ileum and colon & $19(58 \%)$ & $24(69 \%)$ & $19(49 \%)$ & $62(58 \%)$ \\
\hline Colon only & $11(33 \%)$ & $7(20 \%)$ & $14(36 \%)$ & $32(30 \%)$ \\
\hline \multicolumn{5}{|l|}{ Previous TNF antagonist use } \\
\hline One antagonist & $9(27 \%)$ & $8(23 \%)$ & $9(23 \%)$ & $26(24 \%)$ \\
\hline Two antagonists & $16(48 \%)$ & $18(51 \%)$ & $22(56 \%)$ & $56(52 \%)$ \\
\hline $\begin{array}{l}\text { Three or more } \\
\text { antagonists }\end{array}$ & $6(18 \%)$ & $7(20 \%)$ & $5(13 \%)$ & $18(17 \%)$ \\
\hline
\end{tabular}

Data are mean (SD), $\mathrm{n}(\%)$, or median (IQR). CDAI=Crohn's Disease Activity Index. CDEIS=Crohn's Disease Endoscopic Index of Severity. $\mathrm{CRP}=\mathrm{C}$-reactive protein. $\mathrm{TNF}=$ tumour necrosis factor.

Table 1: Summary baseline demographics and disease characteristics of patients who entered study Period 2 (by original treatment group) 
remission at week 12 and were in clinical remission at week 26, and one was included in a deviation from the protocol, despite not achieving clinical remission at the

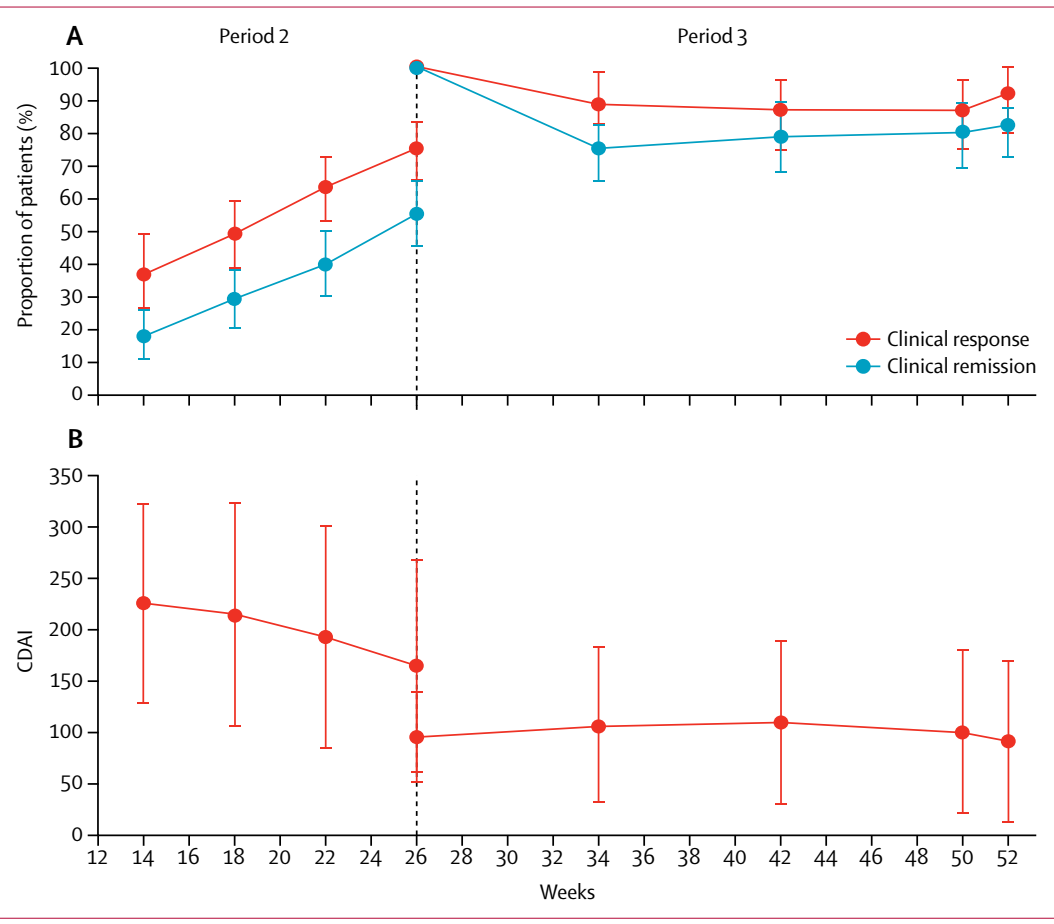

Figure 2: Time course of clinical response and clinical remission ( $A$ ) and CDAI (B) in Periods 2 and 3 Data are from the efficacy analysis population ( $n=101$ in Period 2, $n=62$ in Period 3). We used last observation carried forward for missing values and stratified Cochran-Mantel-Haenszel tests. For clinical response and remission, the error bars are $95 \% \mathrm{Cls}$; for CDAl, results are mean CDAI and SDs are shown with the error bars. CDAl=Crohn's Disease Activity Index.

\begin{tabular}{l} 
Placebo \\
\cline { 3 - 4 }
\end{tabular}

end of Period 2 (figure 1). Eight patients discontinued subcutaneous risankizumab during Period 3 (figure 1). The remaining 54 patients completed 52 weeks of treatment.

During the maintenance phase, the proportion of patients in clinical remission declined slightly by week 34 but was then stable to the end of the study (figure 2A). At week 52, 44 (71\%) of 62 patients were in clinical remission and 50 (81\%) had clinical response (table 2). The proportions of patients in clinical remission and achieving clinical response were similar across the three groups; however, rates of endoscopic remission, endoscopic response, and mucosal healing were highest in patients who had been treated with the $600 \mathrm{mg}$ dose of risankizumab in Period 1 (table 2). Mean CDAI was stable through Period 3 (figure 2B).

At week 52, 18 patients were in deep remission, including five $(83 \%)$ of the six patients who had been in deep remission at week 12. During Periods 2 and 3, four of these patients maintained clinical remission at all visits, another patient maintained remission despite a brief increase in CDAI to 154.5 during Period 2, and the final patient showed fluctuating CDAI and lost remission at the last visit (appendix p 5). All patients who were in deep remission at week 12 had a steep decrease in CDAI during Period 1 (appendix p 5).

C-reactive protein, faecal calprotectin, and faecal lactoferrin concentrations were all reduced during Period 2 in patients receiving $600 \mathrm{mg}$ risankizumab (figure 3). These reductions were maintained to week 52 in patients who entered Period 3 (figure 3).

Safety results for the double-blind, randomised treatment period have been reported previously. ${ }^{21}$ The frequencies of treatment-emergent adverse events per 100 patient-years of exposure to risankizumab for the individual periods are shown in table 3 . No new safety signals were identified in Periods 2 and 3 compared with Period 1. The most frequent treatment-emergent adverse events were arthralgia (25 [22\%] of 115 patients), headache (23 [20\%]), abdominal pain (21 [18\%]), nasopharyngitis (18 [16\%]), nausea (18 [16\%]), and pyrexia (15 [13\%]). Most treatment-emergent adverse events were mild or moderate in severity and were considered by the investigator to be unrelated to study treatment. Discontinuation of risankizumab because of a treatment-emergent adverse event occurred in one (1\%) patient during Period 2 (condition aggravated) and two (3\%) patients during Period 3 (both for worsening of Crohn's disease, one of which was recorded as a serious adverse event). Serious adverse events were experienced by 11 (11\%) patients in Period 2 and seven (11\%) patients in Period 3; those occurring in two or more patients in either period were worsening of Crohn's disease (three patients) and intestinal obstruction (four patients), all during Period 2.

Serious infections were reported in five $(4 \%)$ patients during treatment with risankizumab; each serious infection (anal abscess, appendicitis, incision site 
abscess, osteomyelitis, and pneumonia) was observed in individual patients (appendix p 6). Hepatic disorder or drug-induced liver injury adverse events were reported in seven (6\%) patients treated with risankizumab; all adverse events were grade 1 or 2 , and none met the criteria for Hy's law. No systemic or anaphylactic reactions, neoplasia, or clinically meaningful changes in vital signs were observed in patients treated with risankizumab in any study period, and there were no consistent trends observed in clinical laboratory evaluations.

Post-hoc logistic regression analysis of the population treated in Period 3, using Period 1 treatment group assignment as a classifier did not identify any baseline factors (CDAI, duration of disease, disease location [ileal or colonic], abdominal pain, stool frequency, corticosteroids use, TNF antagonist use, or the presence of draining fistulas) predictive of week 52 clinical remission or response (data not shown). After the step 0 of the intercept entered, no additional effects met the 0.05 significance level for entry into the model.

The mean trough concentrations of risankizumab in patients who received $600 \mathrm{mg}$ intravenous risankizumab during Period $2(35.5 \mu \mathrm{g} / \mathrm{mL})$ were similar to those observed in patients who received $600 \mathrm{mg}$ intravenous risankizumab during Period $1(34 \cdot 2 \mu \mathrm{g} / \mathrm{mL})$. Consistent with the reduction in dose to $180 \mathrm{mg}$ subcutaneous risankizumab every 8 weeks in patients who continued to Period 3, risankizumab concentrations decreased considerably from week 34 and attained near-steadystate levels by week 42 (roughly $4 \mu \mathrm{g} / \mathrm{mL}$ ). Among patients with pharmacokinetic data at week 50, risankizumab median trough plasma concentrations were not substantially different between patients in clinical remission $(\mathrm{n}=37 ; 3.53 \mu \mathrm{g} / \mathrm{mL})$ and those who continued to experience active disease $(n=9$; $3.27 \mu \mathrm{g} / \mathrm{mL}$ ). Median trough plasma concentrations at week 50 were also similar between participants who were receiving a stable dose of immunomodulators (azathioprine, mercaptopurine, or methotrexate; $n=23$; median $3.7 \mu \mathrm{g} / \mathrm{mL})$ versus those who were not $(\mathrm{n}=29$; median $2.9 \mu \mathrm{g} / \mathrm{mL}$ ). Of the patients who entered the washout phase during Period $2(n=6)$, median trough plasma concentrations of risankizumab $(3.5 \mu \mathrm{g} / \mathrm{mL})$ at week 50 were similar to those who had received $600 \mathrm{mg}$ risankizumab during Period $2(\mathrm{n}=46 ; 3 \cdot 3 \mu \mathrm{g} / \mathrm{mL})$.

Treatment-emergent anti-drug antibodies were observed in nine $(8 \%)$ of 108 patients who received at least one dose of risankizumab and had assessable samples at baseline and after treatment initiation. The time to anti-drug antibody positivity ranged between 12 and 18 weeks after the start of treatment, and most anti-drug antibody responses were transient with low titre values. None of the patients positive for anti-drug antibodies had neutralising antibodies and no association was detected between the presence of anti-drug antibodies and risankizumab plasma concentrations.

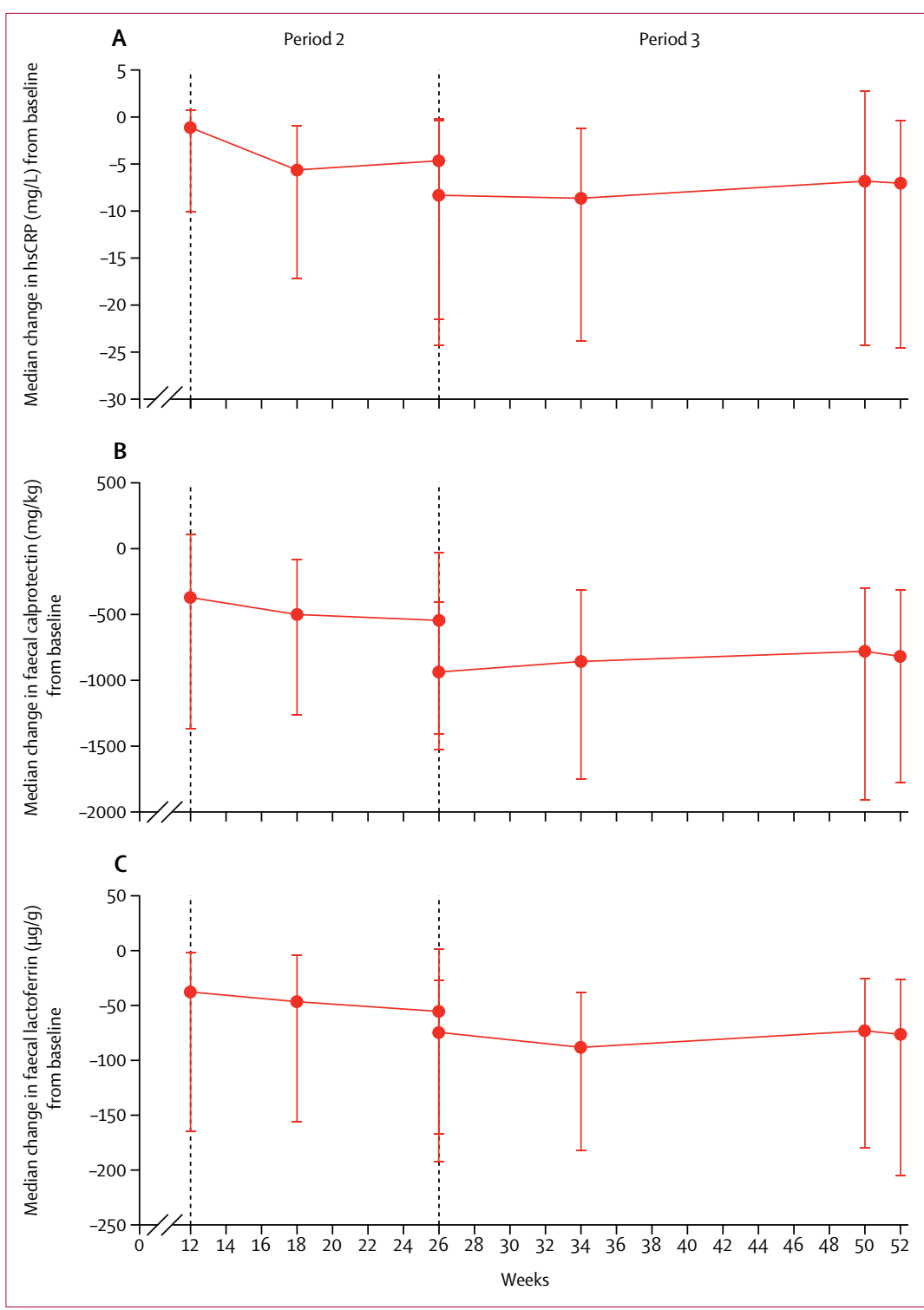

Figure 3: Median change in CRP, FCP, and LF in Periods $2(n=101)$ and $3(n=62)$

We used the efficacy analysis population for this analysis, using last observation carried forward for missing values and stratified Cochran-Mantel-Haenszel tests. Median change from Period 1 baseline values and IQR are reported. hs $C R P=$ high-sensitivity $C$-reactive protein. $F C P=$ faecal calprotecin. $L F=$ faecal lactoferrin.

\section{Discussion}

As previously reported, blockade of interleukin 23 p19 with risankizumab was superior to placebo in achieving clinical remission and clinical response in patients with moderate to severe, treatment-refractory, Crohn's disease. ${ }^{21}$ All efficacy outcomes at week 12 favoured the $600 \mathrm{mg}$ risankizumab dose, suggesting that the higher dose is superior to the lower dose $(200 \mathrm{mg})$ as induction therapy. ${ }^{21}$

For patients who did not achieve deep remission at week 12, switching to treatment with open-label intravenous risankizumab $(600 \mathrm{mg})$ resulted in greater clinical remission rates than those observed in the same 


\begin{tabular}{|lccc}
\hline & Period 2 (n=101) & Period 3 (n=62) & Periods 1-3 (n=115) \\
\hline Patient-years & $28 \cdot 8$ & $43 \cdot 3$ & $109 \cdot 1$ \\
\hline Any adverse event & $267(927 \cdot 1)$ & $165(381 \cdot 1)$ & $719(659 \cdot 0)$ \\
Severe & $19(66 \cdot 0)$ & $6(13 \cdot 9)$ & $36(33 \cdot 0)$ \\
Possibly drug-related* & $28(97 \cdot 2)$ & $23(53 \cdot 1)$ & $81(74 \cdot 2)$ \\
Leading to discontinuation of drug & $1(3 \cdot 5)$ & $2(4 \cdot 6)$ & $10(9 \cdot 2)$ \\
Leading to death & 0 & 0 & 0 \\
Serious adverse event & $18(62 \cdot 5)$ & $9(20 \cdot 8)$ & $46(42 \cdot 2)$ \\
Infections & $37(128 \cdot 5)$ & $32(73 \cdot 9)$ & $107(98 \cdot 1)$ \\
Serious infections & $1(3 \cdot 5)$ & $1(2 \cdot 3)$ & $5(4 \cdot 6)$ \\
Opportunistic infections & 0 & 0 & $3(2 \cdot 7)$ \\
Tuberculosis & 0 & 0 & $1(0 \cdot 9)$ \\
Fungal & $3(10 \cdot 4)$ & 0 & $8(7 \cdot 3)$ \\
Malignancies & 0 & 0 & 0 \\
Infusion-related reaction & $3(10 \cdot 4)$ & NA & $4(3 \cdot 7)$ \\
Drug-induced liver injury or hepatic disorder† & $5(17 \cdot 4)$ & $6(13 \cdot 9)$ & $13(11 \cdot 9)$ \\
Cardiac arrhythmias & $1(3 \cdot 5)$ & 0 & $1(0 \cdot 9)$ \\
Systemic hypersensitivity or anaphylactic & $20(69 \cdot 4)$ & $12(27 \cdot 7)$ & $49(44 \cdot 9)$ \\
reactions & & & \\
Depression, suicidal ideation and behaviour & $1(3 \cdot 5)$ & $2(4 \cdot 6)$ & $5(4 \cdot 6)$ \\
\hline
\end{tabular}

Data are number of events (events per 100 patient-years). Treatment-emergent adverse events are defined as events that begin or worsen either on or after the first dose of the study drug, and within 105 days after the last dose of the study drug. NA=not applicable. *Assessed by study investigator. + All adverse events were grade 1 or 2 , and none met the criteria for Hy's law.

Table 3: Treatment-emergent adverse events per 100 patient-years, in patients who received at least one dose of study drug during the indicated study period

group of patients at week 12 . In patients who switched from placebo to $600 \mathrm{mg}$ risankizumab, the proportion of patients in clinical remission increased from $18 \%$ to $55 \%$, corroborating the efficacy results from the initial blinded induction period. ${ }^{21}$ Dose escalation from $200 \mathrm{mg}$ risankizumab in Period 1 to $600 \mathrm{mg}$ risankizumab in Period 2 more than doubled the percentage of patients in clinical remission, which is supportive of the dose-dependent efficacy noted in the double-blind phase of the study. Among patients originally treated with $600 \mathrm{mg}$ risankizumab, extended treatment duration at the same dose was associated with an increase in clinical remission rates, suggesting that some patients might benefit from extended induction treatment or from a shorter induction duration with a higher dose. Bearing in mind that most patients in this study had failed therapy with one or more TNF antagonists, this finding is consistent with results from trials of other biological therapies that suggest that patients with extensive previous treatment with TNF antagonists might take longer to achieve remission. ${ }^{1,22}$ All patients in deep remission at week 12 who received no treatment in Period 2 remained in clinical remission at week 26. Larger phase 3 trials are ongoing, which will allow the assessment of predictors of clinical remission.

The data suggest that open-label $180 \mathrm{mg}$ subcutaneous risankizumab is effective in maintaining clinical remission up to week 52 in patients who were in clinical remission at week 26 , including those who had achieved deep remission at week 12 and were not treated during weeks 14-26. The rate of deep remission was greater at week 52 than it was at week 12, supporting the hypothesis that endoscopic remission commonly follows clinical remission with increasing duration of treatment. The lack of correlation between risankizumab plasma levels and remission status at week 52 could be attributed to the variability between participants in sensitivity for the drug effect, and the fact that a single maintenance dose level was assessed in the study. Therefore, at the steady-state exposures achieved with the $180 \mathrm{mg}$ subcutaneous maintenance dose, some patients might not have a sustained response because they have lower sensitivity to the drug compared with patients who have sustained responses, despite comparable plasma exposures. It remains to be seen whether increased maintenance doses can increase the proportion of patients who maintain remission. The highest rates of endoscopic remission, endoscopic response, and mucosal healing at week 52 were in patients who were originally randomised to the risankizumab $600 \mathrm{mg}$ group, suggesting that higher initial drug exposure increases the endoscopic resolution of disease activity.

Overall, risankizumab was well tolerated with no new safety signals detected during extended intravenous dosing or during the subcutaneous treatment period. Serious adverse events reported in two or more patients treated with risankizumab were primarily gastrointestinal in nature and might reflect underlying disease.

The study had some limitations. First, a relatively small number of patients was assessed, preventing robust subgroup analysis of clinically relevant populations. In particular, the inclusion of a small number of TNF antagonist-naive patients precludes any comment on the relative efficacy of risankizumab to those without previous TNF antagonist exposure. Second, the endoscopic outcomes used in the study have not been fully validated. However, this is a general limitation of studies in this field and the definitions used are consistent with expert panel recommendations. ${ }^{25,26}$ Third, use of open-label dosing might have increased the perceived efficacy of risankizumab therapy for subjective endpoints such as the CDAI. Specifically, the absence of a control group in the extension study prevents us from drawing strong conclusions regarding the efficacy of risankizumab as a maintenance therapy. Finally, a plateauing of the dose-response was not shown in the double-blinded 12-week induction study, ${ }^{21}$ suggesting that a dose higher than $600 \mathrm{mg}$ could increase efficacy. Furthermore, no subcutaneous dose ranging was done during the maintenance phase, and therefore the optimal dose for the maintenance of clinical remission was not identified.

In conclusion, these results suggest that extended treatment with $600 \mathrm{mg}$ intravenous risankizumab in patients with moderate to severe, treatment-refractory Crohn's disease who were not in deep remission at 
week 12 , increases clinical response and remission rates at week 26. The results also suggest that open-label $180 \mathrm{mg}$ subcutaneous risankizumab is efficacious in the maintenance of clinical remission at week 52 .

\section{Contributors}

The initial draft was prepared by professional medical writers under the direction of BGF. All authors approved the manuscript for submission and vouch for the veracity and completeness of the data and the fidelity of the study to the protocol. BGF, JP, AK, GD'H, WJS, SJP, SV, DBH, and WOB contributed to the study design. BGF, JP, MF, AK, GRD'H, WJS, EL, MFN, DF, OD, US, K-JK, and CS contributed to data collection. All authors contributed to data analysis, data interpretation, and writing and review of the manuscript.

\section{Declaration of interests}

BGF reports personal fees from Ablynx, ActoGeniX, Akros, Albireo Pharma, Allergan, Avaxia Biologics Inc, Avir Pharma, Atlantic Pharma, Baxter Healthcare Corporation, Biogen Idec, Boehringer Ingelheim, Calypso Biotech, Celgene, Elan/Biogen, enGene, Ferring, Galapagos, Genentech/Roche, GiCare Pharma, Gilead, Given Imaging, GlaxoSmithKline, Inception IBD Inc, Ironwood, Japan Tobacco Company, Kyowa Hakko Kirin Co Ltd, Lexicon, Eli Lilly, Lycera Biotech, Merck, Mesoblast Pharma, Millennium, Nestles, Novo Nordisk, Novartis, NextBiotix, Progenity, Prometheus Therapeutics \& Diagnostics, Protagonist, Salix, Shire, Sigmoid Pharma, Synergy Pharma, Teva Pharma, TiGenix, Tillotts, UCB, Vertex, VHsquared, Vivelix Pharma, Wyeth, Zealand, and Zyngenia; grants from Sanofi; grants and personal fees from AbbVie, Amgen, AstraZeneca, Bristol-Myers Squibb, Janssen Biotech/Centocor, Johnson and Johnson/Janssen, Pfizer, Receptos, and Takeda, outside the submitted work; and is a Senior Scientific Director for Robarts Clinical Trials Inc Western University, London, ON, Canada. JP is an advisor for AbbVie, Amgen, Boehringer Ingelheim, Genentech/Roche, Janssen, Merck Sharp Dohme (MSD), Novartis, Oppilan, Pfizer, Takeda, Theravance, and TiGenix. MF is an advisor for AbbVie, Boehringer Ingelheim, Ferring, Janssen, Mitsubishi Tanabe, MSD, Pfizer, and Takeda; has received research grants from Janssen and Takeda; and has received speaker fees from AbbVie, Boehringer Ingelheim, Chiesi, Ferring, Janssen, Lamepro, Mitsubishi Tanabe, MSD, Pfizer, Tramedico, Tillotts, and Zeria. AK is an advisor for Boehringer Ingelheim, Ferring, Genentech, GlaxoSmithKline, Gilead, Hospira, Janssen/Johnson \& Johnson, Pfizer, and VHsquared. GRD'H is an advisor for AbbVie, Ablynx, Amakem, Amgen, AM Pharma, Avaxia, Biogen, Bristol-Myers Squibb, Boehringer Ingelheim, Celgene/Receptos, Celltrion, Cosmo, Covidien/Medtronics, Ferring, Dr Falk Pharma, Eli Lilly, enGene, Galapagos, Genentech/Roche, Gilead, GlaxoSmithKline, Immunic, Johnson and Johnson, Lycera, Medimetrics, Millennium/Takeda, Mitsubishi Pharma, MSD, Mundipharma, Nextbiotics, Novo Nordisk, Otsuka, Pfizer/Hospira, Prometheus Laboratories/Nestle, Protagonist, Robarts Clinical Trials, Salix, Samsung Bioepis, Sandoz, SetPoint, Shire, Teva, TiGenix, Tillotts, Topivert, Versant, and Vifor; has received speaker fees from AbbVie, Biogen, Ferring, Johnson and Johnson, MSD, Mundipharma, Norgine, Pfizer, Samsung Bioepis, Shire, Millennium/ Takeda, Tillotts, and Vifor; and is a Scientific Advisor for Robarts Clinical Trials Inc, Western University, London, ON, Canada. WJS reports consulting fees from AbbVie, Akros Pharma, Allergan, Ambrx Inc., Amgen, Ardelyx, Arena Pharmaceuticals, Atlantic Pharmaceuticals, Avaxia, Biogen, Boehringer Ingelheim, Bristol-Myers Squibb, Celgene, Conatus, Cosmo Technologies, Escalier Biosciences, Ferring, Ferring Research Institute, Forward Pharma, Galapagos, Genentech, Gilead Sciences, Immune Pharmaceuticals, Index Pharmaceuticals, Janssen, Kyowa Hakko Kirin Pharma, Eli Lilly, Medimmune, Mesoblast, Miraca Life Sciences, Nivalis Therapeutics, Novartis, Nutrition Science Partners, Oppilan Pharma, Otsuka, Palatin, Paul Hastings, Pfizer, Precision IBD, Progenity, Prometheus Laboratories, Qu Biologics, Regeneron, Ritter Pharmaceuticals, Robarts Clinical Trials (owned by University of Western Ontario), Salix, Seattle Genetics, Seres Therapeutics, Shire, Sigmoid Biotechnologies, Takeda, Theradiag, Theravance, TiGenix, Tillotts Pharma, UCB Pharma, Vascular Biogenics, Vivelix; research grants from Atlantic Healthcare
Limited, Amgen, Genentech, Gilead Sciences, AbbVie, Janssen, Takeda, Eli Lilly, Celgene/Receptos; payments for lectures or speakers bureau from AbbVie, Janssen, Takeda; and holds stock or stock options in Escalier Biosciences, Oppilan Pharma, Precision IBD, Progenity, Ritter Pharmaceuticals; and is a Scientific Advisor for Robarts Clinical Trials Inc, Western University, London, Ontario, Canada. EL reports research grants from Takeda and Pfizer; educational grants from AbbVie, MSD, and Takeda; speaker fees from Abbott, AbbVie, AstraZeneca, Ferring, MSD, Chiesi, Dr Falk Pharma, Takeda, Hospira, Janssen, Pfizer; and has provided consultancy to Abbott, AbbVie, Ferring, MSD, Mitsubishi Pharma, Takeda, Celltrion, Celgene, Hospira, and Janssen. MFN reports personal fees from Boehringer Ingelheim, Index Pharmaceuticals AB, Janssen, MSD, Pentax Europe, PPM Services, Shire, and Takeda. US reports research grants from Boehringer Ingelheim, Celltrion, Gilead, InDex Pharmaceuticals, Pfizer, Janssen, Roche, and Takeda; and non-financial support for a scientific meeting invitation from Takeda. CS reports receiving research grants from AbbVie, Takeda, and Warner Chilcott; has provided consultancy to AbbVie, Dr Falk Pharma, Janssen, Takeda, and Warner Chilcott; and receiving speaker fees from AbbVie, Dr Falk Pharma, MSD, Takeda, and Warner Chilcott. SJP, IH, AS, SV, $\mathrm{DBH}$, and WOB report being employed by Boehringer Ingelheim. AMR, JZ, and MM report being employed by AbbVie Inc; AAO and KW are employed by AbbVie Inc, and are shareholders of AbbVie Inc. DF, OD, and K-JK declare no competing interests.

\section{Acknowledgments}

Editorial assistance was provided by Esther Race and Leigh Church of SuccinctChoice Medical Communications (London, UK) and was funded by Boehringer Ingelheim (Ingelheim, Germany). All authors had full access to the study data, input into the content of the manuscript, and approved the final version for submission. We thank all the patients and study investigators who participated in this study.

\section{References}

1 Gomollon F, Dignass A, Annese V, et al. 3rd European evidence-based consensus on the diagnosis and management of Crohn's disease 2016: Part 1: diagnosis and medical management. J Crohns Colitis 2017; 11: 3-25.

2 Baumgart DC, Sandborn WJ. Crohn's disease. Lancet 2012; 380: 1590-605.

3 Terdiman JP, Gruss CB, Heidelbaugh JI, Sultan S, Falck-Ytter YT American Gastroenterological Association Institute guideline on the use of thiopurines, methotrexate, and anti-TNF- $\alpha$ biologic drugs for the induction and maintenance of remission in inflammatory Crohn's disease. Gastroenterology 2013; 145: 1459-63.

4 Peyrin-Biroulet L, Deltenre P, de Suray N, Branche J, Sandborn WJ, Colombel JF. Efficacy and safety of tumor necrosis factor antagonists in Crohn's disease: meta-analysis of placebo-controlled trials. Clin Gastroenterol Hepatol 2008; 6: 644-53.

5 Gordon JP, McEwan PC, Maguire A, Sugrue DM, Puelles I. Characterizing unmet medical need and the potential role of new biologic treatment options in patients with ulcerative colitis and Crohn's disease: a systematic review and clinician surveys. Eur J Gastroenterol Hepatol 2015; 27: 804-12.

6 Lichtenstein GR, Abreu MT, Cohen R, Tremaine W. American Gastroenterological Association Institute medical position statement on corticosteroids, immunomodulators, and infliximab in inflammatory bowel disease. Gastroenterology 2006; 130: 935-39.

7 Duerr RH, Taylor KD, Brant SR, et al. A genome-wide association study identifies $I L 23 R$ as an inflammatory bowel disease gene. Science 2006; 314: 1461-63.

8 Geremia A, Arancibia-Carcamo CV, Fleming MP, et al. IL-23-responsive innate lymphoid cells are increased in inflammatory bowel disease. J Exp Med 2011; 208: 1127-33.

9 Uhlig HH, McKenzie BS, Hue S, et al. Differential activity of IL-12 and IL-23 in mucosal and systemic innate immune pathology. Immunity 2006; 25: 309-18.

10 Kullberg MC, Jankovic D, Feng CG, et al. IL-23 plays a key role in Helicobacter hepaticus-induced T cell-dependent colitis. J Exp Med 2006; 203: 2485-94.

11 Gaffen SL, Jain R, Garg AV, Cua DJ. The IL-23-IL-17 immune axis from mechanisms to therapeutic testing. Nat Rev Immunol 2014; 14: 585-600. 
12 Neurath MF. IL-23: a master regulator in Crohn disease. Nat Med 2007; 13: 26-28.

13 Campa M, Mansouri B, Warren R, Menter A. A review of biologic therapies targeting IL-23 and IL-17 for use in moderate-to-severe plaque psoriasis. Dermatol Ther (Heidelb) 2016; 6: 1-12.

14 Gaspari AA, Tyring S. New and emerging biologic therapies for moderate-to-severe plaque psoriasis: mechanistic rationales and recent clinical data for IL-17 and IL-23 inhibitors. Dermatol Ther 2015; 28: 179-93.

15 Feagan BG, Sandborn WJ, Gasink C, et al. Ustekinumab as induction and maintenance therapy for Crohn's disease. N Engl J Med 2016; 375: 1946-60.

16 Sandborn WJ, Gasink C, Gao LL, et al. Ustekinumab induction and maintenance therapy in refractory Crohn's disease. N Engl J Med 2012; 367: 1519-28.

17 Baker KF, Isaacs JD. Novel therapies for immune-mediated inflammatory diseases: what can we learn from their use in rheumatoid arthritis, spondyloarthritis, systemic lupus erythematosus, psoriasis, Crohn's disease and ulcerative colitis? Ann Rheum Dis 2018; 77: 175-87.

18 Hueber W, Sands BE, Lewitzky S, et al. Secukinumab, a human anti-IL-17A monoclonal antibody, for moderate to severe Crohn's disease: unexpected results of a randomised, double-blind placebo-controlled trial. Gut 2012; 61: 1693-700.

19 Targan SR, Feagan B, Vermeire S, et al. A randomized, double-blind, placebo-controlled phase 2 study of brodalumab in patients with moderate-to-severe Crohn's disease. Am J Gastroenterol 2016; 111: 1599-607.
20 Singh S, Kroe-Barrett RR, Canada KA, et al. Selective targeting of the IL23 pathway: Generation and characterization of a novel high-affinity humanized anti-IL23A antibody. MAbs 2015; 7: 778-91.

21 Feagan BG, Sandborn WJ, D'Haens G, et al. Induction therapy with the selective interleukin-23 inhibitor risankizumab in patients with moderate-to-severe Crohn's disease: a randomised, double-blind, placebo-controlled phase 2 study. Lancet 2017; 389: 1699-709.

22 Sands BE, Feagan BG, Rutgeerts P, et al. Effects of vedolizumab induction therapy for patients with Crohn's disease in whom tumor necrosis factor antagonist treatment failed. Gastroenterology 2014; 147: 618-27.

23 Levesque BG, Sandborn WJ, Ruel J, Feagan BG, Sands BE, Colombel JF. Converging goals of treatment of inflammatory bowel disease from clinical trials and practice. Gastroenterology 2015; 148: $37-51$.

24 Krueger JG, Ferris LK, Menter A, et al. Anti-IL-23A mAb BI 655066 for treatment of moderate-to-severe psoriasis: safety, efficacy, pharmacokinetics, and biomarker results of a single-rising-dose, randomized, double-blind, placebo-controlled trial. J Allergy Clin Immunol 2015; 136: 116-24.

25 Vuitton L, Marteau P, Sandborn WJ, et al. IOIBD technical review on endoscopic indices for Crohn's disease clinical trials. Gut 2016; 65: $1447-55$.

26 Novak G, Parker CE, Pai RK, et al. Histologic scoring indices for evaluation of disease activity in Crohn's disease. Cochrane Database Syst Rev 2017; 7: CD012351. 